\title{
Influência da cera de carnaúba no comportamento reológico de misturas usadas na moldagem por injeção em baixa pressão
}

\section{(Influence of carnauba wax in the feedstock rheology used in low-pressure injection molding)}

\author{
P. A. Ourique, R. C. D. Cruz, J. E. Zorzi* \\ Instituto de Materiais Cerâmicos, Universidade de Caxias do Sul, Bom Princípio, RS, Brazil 95765-000 \\ *jezorzi@ucs.br
}

\begin{abstract}
Resumo
A moldagem por injeção em baixa pressão (MIBP) tem algumas vantagens sobre outros métodos de conformação de peças cerâmicas com formas complexas. Dentre as vantagens estão os menores custos na produção de lotes pequenos e médios e o menor desgaste de moldes e equipamento. Neste trabalho, foi estudado o efeito de um dos aditivos orgânicos usados na MIBP, a cera de carnaúba, no comportamento reológico da mistura (feedstock). O pó cerâmico utilizado foi uma alumina, com tamanho de partícula em torno de $0,4 \mu \mathrm{m}$. Com adições de $5 \%$ e $10 \%$ em peso de cera de carnaúba no veículo orgânico a viscosidade diminui significativamente, sendo bem mais acentuada com $10 \%$, enquanto que, acima deste valor (15\%), a viscosidade diminui, mas esta redução é menos pronunciada. Em contrapartida, em feedstocks sem a cera de carnaúba ( $0 \%$ ), a viscosidade é tão elevada que praticamente inviabiliza a injeção em baixas pressões.
\end{abstract}

Palavras-chave: moldagem por injeção em baixa pressão, alumina, cera de carnaúba, reologia.

\section{Abstract}

The low-pressure injection molding (LPIM) has some advantages over other methods of forming ceramic parts with complex shapes. Among the advantages are the lower costs in the production of small and medium lots and less wear of molds and equipment. In this work, the effect of an organic additive used in LPIM been studied, carnauba wax, on the rheology of the mixture (feedstock). The ceramic powder used was alumina with particle size of about 0.4 micrometers. With additions of 5 wt $\%$ and 10 wt $\%$ of carnauba wax in the organic vehicle, viscosity decreases significantly, being much more pronounced at 10\%, while above this value (15\%), the viscosity decrease, but this reduction is less pronounced. In contrast, in feedstock without carnauba wax ( $0 \%)$, the viscosity is so high that the injection almost impossible at low pressures.

Keywords: low-pressure injection molding, alumina, carnauba wax, rheology.

\section{INTRODUÇÃO}

A moldagem por injeção de baixa pressão (MIBP) foi desenvolvida por Gribovski há aproximadamente 70 anos [1]. Este método de conformação tem se mostrado adequado para a produção de pequenos e médios lotes de peças cerâmicas com formatos complexos, envolvendo custos de fabricação consideravelmente mais baixos, desgaste menor da injetora e dos moldes, se comparado com a moldagem por injeção de alta pressão [1-3]. A mistura de pós cerâmicos e veículo orgânico (VO) usada na MIBP deve ser otimizada para possuir alta fluidez, mantendo o teor de sólidos o mais elevado possível. Os componentes do VO podem alterar o comportamento reológico aumentando ou diminuindo a viscosidade da mistura dependendo da sua composição química. O VO usado na MIBP é formado principalmente por ceras. Embora o VO não seja o componente principal na moldagem por injeção, ele é responsável pelo sucesso no processo de conformação de peças cerâmicas $[3,4]$.
As ceras em geral atuam como polímeros de cadeias curtas. Possuem baixa temperatura de fusão e baixa viscosidade. A parafina é a cera mais utilizada como componente principal na MIBP. Outras ceras utilizadas são a cera de polietileno, cera de carnaúba, parafina microcristalina, cera de abelha, entre outras. Além das ceras, são usados agentes modificadores de superfície como os ácidos carboxílicos, por exemplo [3, 4]. A cera de carnaúba é uma cera vegetal brasileira, e possui como característica ser dura e quebradiça, quando comparada às demais. Possui uma temperatura de fusão entre 80 e $87^{\circ} \mathrm{C}$ [3]. Esta cera se forma nas folhas e frutos das palmeiras de carnaúba (Copernicia cerifera) [5-7]. Sua composição química depende da planta de onde é extraída e consiste em aproximadamente $82-85 \%$ de alquil ésteres de ácidos graxos alifáticos e aromáticos (principalmente o ceril palmitato $\mathrm{C}_{25} \mathrm{H}_{51} \mathrm{COOC}_{30} \mathrm{H}_{61}$ ) $[6,8$, 9], $10-12 \%$ álcoois livres, 5-7\% ácidos graxos livres, $0,3-$ $1 \%$ hidrocarbonetos e $0,4-0,5 \%$ de outros componentes [4, 7-11]. Na MIBP a cera de carnaúba atua como ligante e 
como lubrificante interno $[5,6]$.

A parafina é um material orgânico apolar, o que dificulta a mistura com os óxidos cerâmicos. Por isso a necessidade de se utilizarem ácidos carboxílicos como agentes dispersantes $[12,13]$. As ceras polares interagem mais fortemente com a superfície dos óxidos do que as apolares, devido à presença dos grupos hidroxila na superfície do pó [1,14]. A adição dos ácidos carboxílicos reduz a viscosidade e aumenta a molhabilidade do pó $[12,15]$. Outro fator importante que pode dificultar uma boa dispersão do pó cerâmico no ácido carboxílico é a presença de aglomerados de pó. Aglomerados de pó aumentam a viscosidade da suspensão, devido ao aprisionamento do meio líquido dentro dos aglomerados, através da redução da fração do volume livre do meio que é necessário para a suspensão fluir [16-18]. Uma formulação para MIBP, já utilizada em trabalhos anteriores [2, 3, 18-22], tem sido utilizada durante muitos anos com relativo sucesso, mas nunca chegou a ser estudada em relação ao efeito da cera de carnaúba sobre a viscosidade desta suspensão. Portanto, neste trabalho, a cera de carnaúba foi analisada com relação ao seu comportamento reológico em diferentes concentrações no feedstock. O objetivo deste estudo é reduzir a viscosidade, para futuramente obter uma nova formulação, com maior concentração de sólidos e que ainda possa ser injetada a baixas pressões, sem a perda de fluidez e sem a formação de defeitos. Foi utilizado pó de alumina desaglomerado com proporções de 0 a $2,1 \%$ em peso de cera de carnaúba na massa total do feedstock, que corresponde de 0 a $15 \%$ em peso de cera de carnaúba no VO.

\section{MATERIAIS E MÉTODOS}

O pó cerâmico utilizado neste trabalho foi $\alpha$-alumina calcinada A-1000SG (Almatis Inc., Leetsdale, PA, EUA). A composição é $\mathrm{Al}_{2} \mathrm{O}_{3}-99,8 \%, \mathrm{Na}_{2} \mathrm{O}-0,07 \%, \mathrm{SiO}_{2}-$ $0,03 \%, \mathrm{Fe}_{2} \mathrm{O}_{3}-0,02 \%, \mathrm{CaO}-0,02 \%$ e $\mathrm{MgO}-0,04 \%$, área superficial do pó de $8,4 \mathrm{~m}^{2} / \mathrm{g}$ e tamanho médio de partícula de $0,4 \mu \mathrm{m}$ (dados do fabricante).

Para destruir os aglomerados do pó, a alumina foi desagregada em água em um moinho de esferas com revestimento do moinho e esferas de alumina [18]. O tempo de desagregação foi de $24 \mathrm{~h}$, a $32 \mathrm{rpm}$, com $80 \%$ do volume do moinho ocupado por esferas, pó e água destilada e deionizada [18]. A seguir o pó foi seco a $100{ }^{\circ} \mathrm{C} / 24 \mathrm{~h} \mathrm{em}$ estufa e novamente desaglomerado, desta vez manualmente em almofariz.
A mistura de alumina e VO para a injeção foi preparada diretamente no tanque da injetora (Peltsman MIGL-33, Minneapolis, MN, EUA), com $86 \%$ em peso de alumina e $14 \%$ em peso de VO ( 41,7\%vol.) [18]. O veículo orgânico empregado é composto por cera de parafina, cera de carnaúba ( 0 a 15\% em peso do VO), cera de polietileno e ácido oleico, conforme Tabela I [2, 3, 18-22],

Após ter sido seco a $120{ }^{\circ} \mathrm{C} / 3 \mathrm{~h}$, o pó cerâmico foi adicionado no $\mathrm{VO}$ fundido [18]. O VO foi fundido dentro do tanque da própria injetora previamente aquecida a $90{ }^{\circ} \mathrm{C}$ e o pó ainda quente foi adicionado lentamente em alíquotas sob agitação constante.

O ligante principal usado foi a parafina (120/125-3 Petrobras, BR), que possui o ponto de fusão entre $49-52{ }^{\circ} \mathrm{C}$. Outros componentes do veículo orgânico foram o ácido oleico (Synth, BR), que possui temperatura de fusão de $16^{\circ} \mathrm{C}$, a cera de polietileno (Ipiranga Petroquímica, BR), com temperatura de fusão em torno de $90^{\circ} \mathrm{C}$, e a cera de carnaúba (do tipo III), com temperatura de fusão entre $80-87^{\circ} \mathrm{C}$. A composição da mistura de pó cerâmico com VO pode ser vista na Tabela I (em peso e em volume) e na Tabela II (cera de carnaúba). Na Tabela II estão relacionadas as quantidades de cera de carnaúba e parafina, no veículo orgânico (VO) e no feedstock. Partiu-se da referência de $10 \%$ em peso de carnaúba na mistura e, para completar $100 \%$, a ausência ou excesso de cera de carnaúba foi compensada pela adição ou remoção de parafina.

A mistura de alumina e VO foi injetada em um molde metálico a $90{ }^{\circ} \mathrm{C}$ e $400 \mathrm{kPa}$ de pressão, mantendo esta pressão por $12 \mathrm{~s}$. As amostras injetadas possuíam formato de disco com $22 \mathrm{~mm}$ de diâmetro e $3 \mathrm{~mm}$ de espessura.

\section{Reologia}

As propriedades reológicas das misturas foram analisadas em um reômetro oscilatório Physica MCR 101 (Anton Paar $\mathrm{GmbH}$, Graz, Áustria), usando o sistema de medida placaplaca PP 25-2, com uma distância entre placas (gap) igual a 0,5 $\mathrm{mm}$. As amostras foram estudadas em uma isoterma a $90{ }^{\circ} \mathrm{C}$.

A viscosidade complexa $\left(\eta^{*}\right)$ bem como os módulos de armazenamento $\left(G^{\prime}\right)$ e perda $\left(G^{\prime \prime}\right)$ foram obtidos por ensaios de varredura de frequência angular $(\omega)$ entre 500 à $0,1 \mathrm{rad} / \mathrm{s}$. A tensão de cisalhamento $(\tau)$ foi mantida constante em 10 Pa para para as amostras com 5\%, 10\% e $15 \%$ m de cera de carnaúba no VO. Para a amostra sem cera de carnaúba $(0 \%)$ a tensão de cisalhamento $(\tau)$ foi mantida constante em $50 \mathrm{~Pa}$. As medidas foram realizadas

Tabela I - Quantidades em peso e em volume de alumina e de aditivos do veículo orgânico para a quantidade de $1,4 \%$ em peso de cera de carnaúba no feedstock.

[Table I - Quantities in weight and volume of alumina and additives in the organic vehicle for the amount of $1.4 \%$ wt of carnauba wax in the feedstock.]

\begin{tabular}{ccccccc}
\hline$\%$ & Alumina & $\begin{array}{c}\text { Cera de } \\
\text { parafina }\end{array}$ & $\begin{array}{c}\text { Cera de } \\
\text { carnaúba }\end{array}$ & Cera de polietileno & $\begin{array}{c}\text { Ácido } \\
\text { oleico }\end{array}$ & $\%$ \\
\hline peso & 86 & 10,5 & 1,4 & 1,4 & 0,7 & 100 \\
volume & 58,3 & 31,5 & 4,3 & 3,8 & 2,1 & 100 \\
\hline
\end{tabular}


Tabela II - Quantidades de cera de carnaúba e parafina no veículo orgânico e no feedstock (a ausência ou excesso de cera de carnaúba foi completado com parafina).

[Table II - Quantities of paraffin and carnauba wax in the organic vehicle and feedstock (the deficiency or excess of carnauba wax was completed by paraffin wax).]

\begin{tabular}{ccc}
\hline $\begin{array}{c}\text { \% em peso de cera } \\
\text { de carnaúba } \\
\text { (no VO) }\end{array}$ & $\begin{array}{c}\text { \% em peso de } \\
\text { cera de carnaúba } \\
\text { (no feedstock) }\end{array}$ & $\begin{array}{c}\text { \% em peso de } \\
\text { cera de } \\
\text { parafina } \\
\text { (no feedstock) }\end{array}$ \\
\hline 0 & 0,0 & 11,9 \\
5 & 0,7 & 11,2 \\
10 & 1,4 & 10,5 \\
15 & 2,1 & 9,8 \\
\hline
\end{tabular}

a temperatura constante de $90{ }^{\circ} \mathrm{C}$ em atmosfera inerte (gás nitrogênio), dentro da região de viscoelasticidade linear (Fig. 1).

As propriedades de fluxo de um sistema real, especialmente uma mistura de multicomponentes, como é o caso da matéria-prima usada na moldagem por injeção, contem componentes elásticas e viscosas e podem ser medidas por meio de medições de oscilação, se existir uma correlação linear entre a tensão de cisalhamento $\tau(t)$ e a deformação $\gamma(t)$, dada pela equação (A) [23-25].

$$
\tau(\mathrm{t})=\mathrm{G}^{*} \gamma(\mathrm{t})
$$

A parte real $G^{\prime}$, do módulo complexo $G^{*}$, é conhecida como módulo de armazenamento e descreve a parte elástica da energia de deformação. A parte imaginária $G$ ", é denominada módulo de perda e descreve a parte viscosa de energia de deformação, que é dissipada pelo cisalhamento $\left(\tau_{0}\right.$ : amplitude de tensão de cisalhamento; $\gamma_{0}$ : amplitude da deformação), como mostrado na equação (B) [23-25].

$$
\mathrm{G}^{*}=\mathrm{G}^{\prime}+\mathrm{iG} \mathrm{G}^{\prime}=\frac{\tau_{0}}{\gamma_{0}} \cos (\delta)+\mathrm{i} \frac{\tau_{0}}{\gamma_{0}} \operatorname{sen}(\delta)
$$

O ângulo de fase (ou ângulo de perda), $\delta$, representa o tempo de resposta entre a tensão de cisalhamento aplicada e a deformação resultante (para um sólido elástico ideal, $\delta=0^{\circ}$, domínio do comportamento $G$; para um líquido Newtoniano, $\delta=90^{\circ}$, domínio do comportamento $G$ ").

O módulo complexo $G^{*}$, representa a resistência total de uma substância contra uma deformação aplicada; seu valor absoluto é obtido a partir da equação (C) [23-25].

$$
\left|G^{*}\right|=\sqrt{\mathrm{G}^{2}+\mathrm{G}^{\prime 2}}
$$

A viscosidade complexa, $\eta^{*}$, representa a resistência total de uma substância a um cisalhamento dinâmico; seu valor é obtido a partir da equação (D) [23-25],

$$
\left|\eta^{*}\right|=\frac{\tau(\mathrm{t})}{\gamma(\mathrm{t})}=\frac{\left|\mathrm{G}^{*}\right|}{\omega}=\frac{\sqrt{\mathrm{G}^{\prime 2}+\mathrm{G}^{2}}}{\omega}
$$

na qual é a taxa de cisalhamento e $\omega$ é a frequência angular. Sabendo-se quais as variáveis e como cada uma delas afeta o processo de moldagem por injeção em baixa pressão, foram realizadas análises para a caracterização da suspensão, bem como a obtenção das condições ideais de processamento para moldagem por injeção em baixa pressão de alumina submicrométrica.

\section{RESULTADOS E DISCUSSÃO}

Os resultados obtidos nos ensaios para a determinação da região de viscoelasticidade linear da cera de carnaúba são apresentados na Fig. 1. Pode-se perceber que para a maioria dos percentuais de cera de carnaúba, esta região encontra-se acima de $10 \mathrm{~Pa}$, com exceção da amostra com $0 \%$ de cera de carnaúba, motivo pelo qual utilizou-se $50 \mathrm{~Pa}$ nesta amostra.

À medida que aumenta o percentual de cera de carnaúba, o módulo de armazenamento diminui.

As Figs. 2 e 3 apresentam a variação dos módulos de armazenamento $\left(G^{\prime}\right)$ e de perda $\left(G^{\prime \prime}\right)$ em função da frequência angular para diferentes proporções de cera de carnaúba $(0 \%, 5 \%, 10 \%$ e $15 \%$ em peso do VO). O efeito do teor de cera de carnaúba no comportamento reológico é muito mais pronunciado entre $0 \%$ e $10 \%$ de cera no VO, do que entre $10 \%$ e $15 \%$ (Fig. 3). Maiores teores de cera de carnaúba levam a valores menores de $G$ ' e $G$ ".

A Fig. 3 apresenta a média aritmética dos módulos de armazenamento $\left(G^{\prime}\right)$ e de perda $\left(G^{\prime \prime}\right)$ em função do percentual de cera de carnaúba presente no VO. Este gráfico apresenta uma melhor visualização do efeito que a adição de cera de carnaúba exerce sobre os módulos elástico e viscoso. Pode-se perceber que o módulo de armazenamento decai exponencialmente com o aumento do percentual de cera de carnaúba na mistura. Uma possível explicação

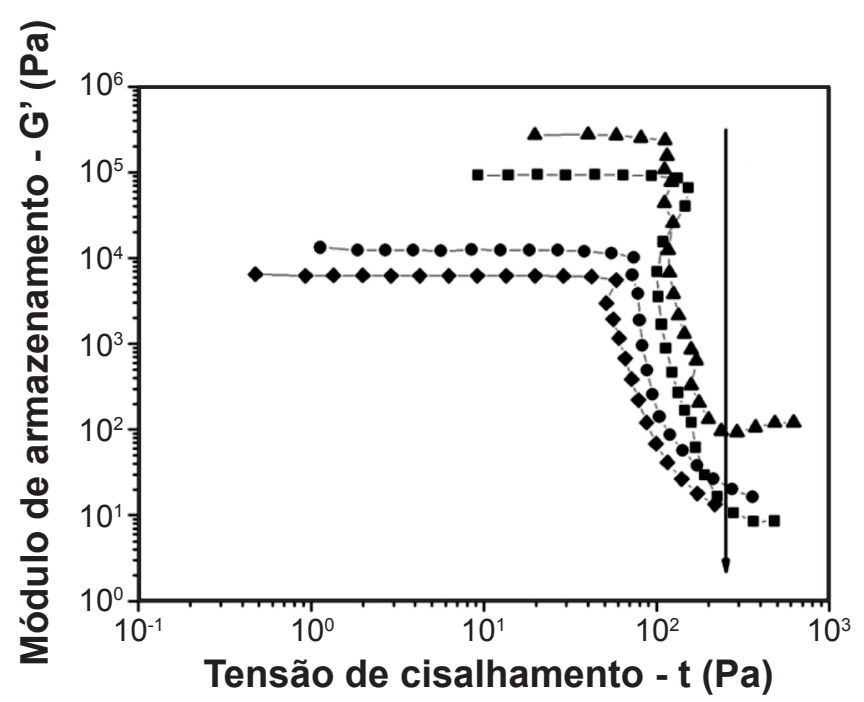

Figura 1: Módulos de armazenamento $\left(G^{\prime}\right)$ versus tensão de cisalhamento $(\tau)$, para percentual em peso de cera de carnaúba no VO de $0 \%(\boldsymbol{\Delta}) ; 5 \%(\mathbf{\square}) ; 10 \%(\bullet)$ e $15 \%(\bullet)$.

[Figure 1: Storage modulus ( $G$ ') versus shear stress $(\tau)$, to the percentage by weight of carnauba wax in the binder of $0 \%(\mathbf{\Delta})$; $5 \%(\mathbf{\square}) ; 10 \%(\bullet)$ and $15 \%(\diamond)$. 


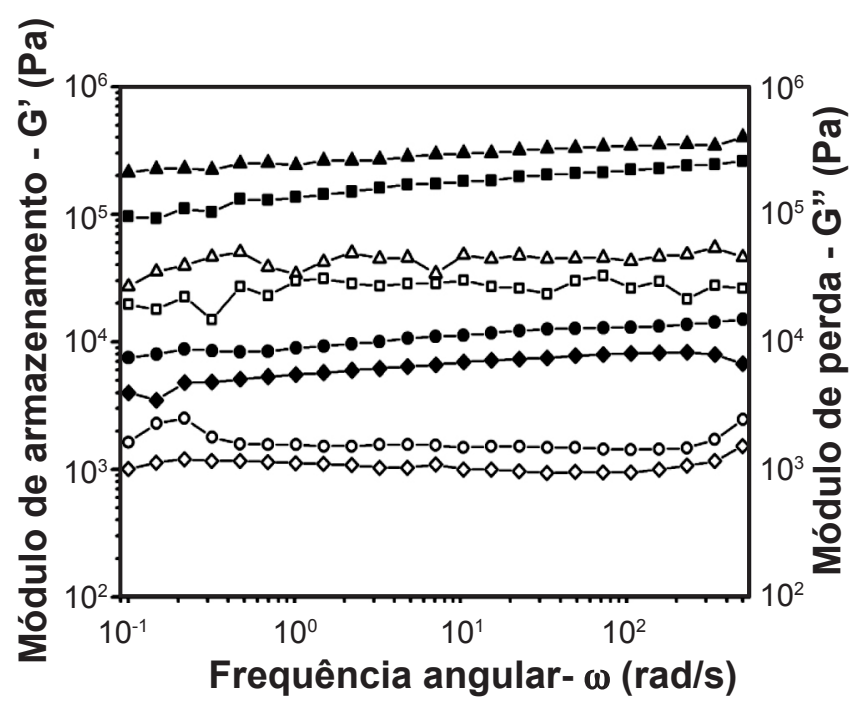

Figura 2: Módulos de armazenamento $\left(G^{\prime}\right)$ (símbolos cheios) e de perda $(G$ ") (símbolos vazados) versus frequência angular $(\omega)$, para percentual em peso de cera de carnaúba no VO de $0 \%(\boldsymbol{\Delta} ; \triangle) ; 5 \%$ $(\mathbf{\square} ; \square) ; 10 \%(\bullet ; \bigcirc)$ e $15 \%(\diamond ; \diamond)$.

[Figure 2: Storage modulus ( $G^{\prime}$ ) (filled symbols) and loss modulus $(G$ ") (open symbols) versus angular frequency $(\omega)$, for percentage by weight of carnauba wax in the binder of $0 \%(\mathbf{\Lambda} ; \triangle) ; 5 \%(\mathbf{\square} ; \square)$; $10 \%(\bullet ;)$ and $15 \%(\diamond)$.]

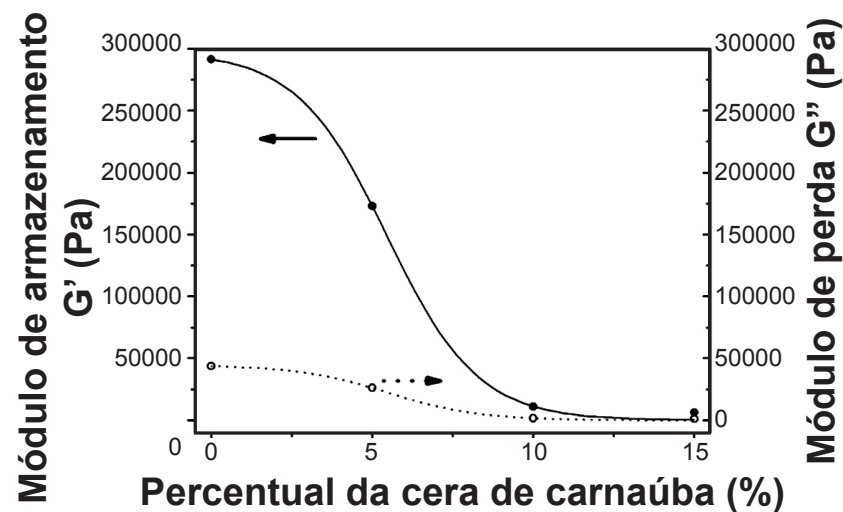

Figura 3: Média aritmética dos módulos de armazenamento $G^{\prime}(\bullet$ e de perda $G$ " $(\mathbf{O})$ versus percentual em peso de cera de carnaúba. [Figure 3: Arithmetic average of the storage modulus $G$ '(-) and loss modulus $G$ “(O) versus percentage by weight of carnauba wax.]

para este fenômeno está no fato da cera de carnaúba atuar como um lubrificante interno da mistura facilitando assim a mobilidade das partículas. Sendo um produto de origem natural, a cera de carnaúba possui muitos componentes (ésteres, ácidos e álcoois, entre outros) e a interação deles com todos os outros ingredientes da mistura ainda não é bem conhecida. Entretanto, fica claro neste resultado que a ausência desta cera leva a valores elevados de $G$ ' e $G$ ”. Na moldagem por injeção em baixas pressões, a viscosidade da mistura não pode ser muito elevada pois, neste caso, não ocorre o total preenchimento da cavidade do molde.

A viscosidade complexa $\left(\eta^{*}\right)$ em função da frequência angular $(\omega)$, para diferentes percentuais em massa de cera de carnaúba nos ligantes, é mostrada na Fig. 4. Aqui também

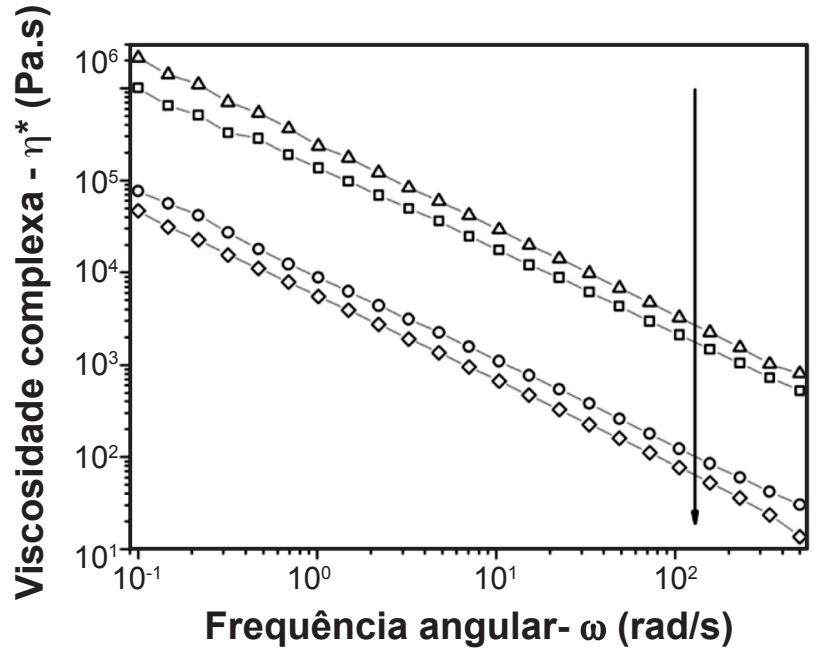

Figura 4: Viscosidade complexa $\left(\eta^{*}\right)$ versus frequência angular $(\omega)$, para percentual em peso de cera de carnaúba no VO de $0 \%$ $(\triangle) ; 5 \%(\square) ; 10 \%(\mathrm{O})$ e $15 \%(\diamond)$.

[Figure 4: Complex viscosity ( $\left.\eta^{*}\right)$ versus angular frequency $(\omega)$, for percentage by weight of carnauba wax in the binder of $0 \%(\Delta)$; $5 \%(\mathrm{a}) ; 10 \%(\mathrm{O})$ and $15 \%(\diamond)$.]

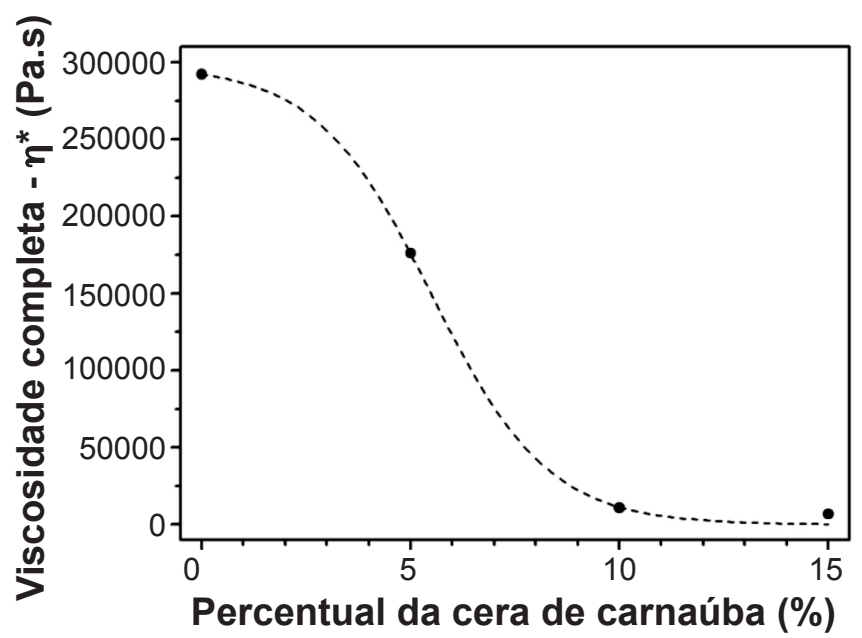

Figura 5: Viscosidade complexa $\left(\eta^{*}\right)$ versus percentual em peso de cera de carnaúba, para frequência angular $(\omega)$ de $10 \mathrm{rad} / \mathrm{s}$.

[Figure 5: Complex viscosity $\left(\eta^{*}\right)$ versus carnauba wax percentage by weight, for angular frequency ( $\omega)$ of $10 \mathrm{rad} / \mathrm{s}$.]

se percebe que a adição de cera de carnaúba diminui a viscosidade da suspensão (seta). Este comportamento já havia sido observado em suspensões para MIBP em medidas de viscosidade aparente [26].

Analisando os gráficos das Figs. 3 e 5, percebe-se que ao aumentar o percentual de cera de carnaúba no feedstock, o decaimento dos módulos de armazenamento (Fig. 3) e a viscosidade complexa (Fig. 5) não apresentam um comportamento linear. No caso da viscosidade complexa, um aumento de $5 \%$ para $10 \%$ no percentual de cera de carnaúba gera uma queda na viscosidade de aproximadamente $16 x$, já ao aumentar o percentual de $10 \%$ para $15 \%$ a queda na viscosidade é de aproximadamente 1,6x. Este comportamento é uma indicação de que percentuais de 
cera de carnaúba maiores que $10 \%$ não geram mudanças significativas na viscosidade da mistura.

Durante este trabalho, foi possível perceber também que misturas preparadas sem a cera de carnaúba (apenas alumina, parafina, cera de polietileno e os ácidos carboxílicos) apresentam uma viscosidade muito elevada, o que torna inviável a injeção em baixas pressões (de até $500 \mathrm{kPa}$ ), sem que ocorra a formação de defeitos, como bolhas ou falhas de preenchimento da cavidade do molde. Em contrapartida, um teor elevado de cera de carnaúba (15\%), uma cera considerada dura e quebradiça, aumenta o risco de perda da peça por lascamento na desmoldagem da peça a verde. Com isso, podese concluir que o benefício decorrente da redução adicional de apenas 1,6x na viscosidade do feedstock, não compensa o risco de rejeição da peça por quebra na desmoldagem, devido ao excesso desta cera.

\section{CONCLUSÕES}

O presente trabalho mostrou o efeito que a presença de cera de carnaúba exerce sobre o comportamento reológico do feedstock destinado à MIBP. No caso da viscosidade complexa, um aumento de $5 \%$ para $10 \%$ no percentual de cera de carnaúba reduz a viscosidade em aproximadamente $16 x$, já ao aumentar o percentual de $10 \%$ para $15 \%$ a redução na viscosidade é de aproximadamente 1,6x. Com isso temos o indicativo de que percentuais de cera de carnaúba maiores que $10 \%$ não geram mudanças significativas na viscosidade da mistura. A redução da viscosidade do feedstock, pelo o aumento do percentual de cera de carnaúba, pode facilitar o preenchimento do molde, diminuindo com isso a possibilidade de surgimento de defeitos no momento da injeção (como bolhas e falhas no preenchimento do molde), o que pode auxiliar na redução do número de peças rejeitadas. Entretanto a cera de carnaúba é uma cera dura e quebradiça e teores muito elevados desta cera podem fragilizar a peça a verde, do que se conclui que, teores em torno de $10 \%$ em peso de cera de carnaúba no $\mathrm{VO}$ (1,4\% em peso no feedstock), estão próximos do ideal para esta formulação. Uma continuidade para este trabalho é o estudo do efeito das demais ceras utilizadas no feedstock (cera de parafina e cera de polietileno) sobre a sua viscosidade. Através desta informação e usando análise estatística é possível otimizar a formulação e a partir daí avaliar a possibilidade de aumentar o teor de sólidos na mistura. A mistura com percentual maior de pó reduz a perda de massa na etapa de remoção dos ligantes, reduzindo com isso a contração da peça sinterizada, o que minimiza a possibilidade do surgimento de defeitos nesta etapa crítica do processamento. Outra possibilidade de continuidade deste trabalho, é refinar este estudo a valores em torno de $10 \%$ em peso de cera de carnaúba até encontrar a fração que seja $100 \%$ ideal e, além disso, para teores mais elevados de cera de carnaúba, comparar os resultados obtidos com o percentual de peças a verde rejeitadas por quebra ou lascamento.

\section{AGRADECIMENTOS}

Os autores agradecem o apoio financeiro da FAPERGS,
CAPES, FINEP e CNPq. Ao LPA da UFRGS e LPol da UCS pelo empréstimo de equipamentos. Ao Prof. Dr. Cláudio A. Perottoni pelas discussões e ao Prof. Dr. Otávio Bianchi, a Matias S. Lunkes, Arthur Susin Neto, Sérgio G. Echeverrigaray pelo auxílio prestado no processamento e nas medidas reológicas.

\section{REFERÊNCIAS}

[1] R. Lenk, Ber. Dtsch. Keram. Ges. 72, 10 (1995) 636-642. [2] J. E. Zorzi, C. A. Perottoni, J. A. H. da Jornada, Ind. Ceram. 23, 1 (2003) 47-49.

[3] J. E. Zorzi, C. A. Perottoni, J. A. H. da Jornada, Cerâmica 50, 315 (2004) 202-208.

[4] S. T. Lin, R. M. German, J. Mater. Sci. 29, 19 (1994) 5207-5212.

[5] I. Majewska-Glabus, L. Zhuang, R. Vetter, J. Duszczyk, J. Mater. Sci. 30, 24 (1995) 6209-6217.

[6] R. Vetter, M. J. Sanders, I. Majewska-Glabus, L. Z. Zhuang, J. Duszczyk, Inter. J. Powder Metal. 30, 1 (1994) 115-124.

[7] B. Elvers, S. Hawkins, Eds., "Ullmann's Encyclopedia of Industrial Chemistry", A28, $5^{\text {th }}$ ed., VHC, Alemanha (1996) pp. 110.

[8] A. H. Warth, "The Chemistry and Technology of Waxes", Reinhold Pub. Corp., $5^{\text {th }}$ ed, A28, VHC, Alemanha (1996) pp. 112.

[9] L. E. Vanderburg, E. A. Wilder, J. Am. Oil Chem. Soc. 44 (1967) 659-662.

[10] L. E. Vanderburg, E. A. Wilder, J. Am. Oil Chem. Soc. 47 (1970) 514-518.

[11] G. Donhowe, O. Fennema, J. Am. Oil Chem. Soc. 70, 9 (1993) 867-873.

[12] R. M. German, "Powder Injection Molding", MPIF, Princeton, NJ, EUA (1990) pp. 3-22.

[13] B. Mutsuddy, R. G. Ford, "Ceramic Injection Molding", Chapman \& Hall, London, UK (1995) pp. 1-9.

[14] R. Lenk, A.Ph. Krivoshchepov. J. Am. Ceram. Soc. 83 (2000) 273-276.

[15] S. Novak, K. Vidovič, M. Sajko, T. Kosmač, J. Eur. Ceram. Soc. 17 (1997) 217-223.

[16] J. Ma, L. C. Lim, J. Eur. Ceram. Soc. 22, 13 (2002) 2197-2208.

[17] D. M. Liu, W. J. Tseng, J. Am. Ceram. Soc. 82, 10 (1999) 2647-2652.

[18] P. A. Ourique, A. Susin Neto, S. G. Echeverrigaray, R. C. D. Cruz, J. E. Zorzi, Cerâmica 59, 349 (2013) 71-77.

[19] I. Krindges, R. Andreola, C. A. Perottoni, J. E. Zorzi, Int. J. Appl. Ceram. Technol. 5, 3 (2008) 243-248.

[20] J. E. Zorzi, C. A. Perottoni, J. A. H. da Jornada, J. Mater. Sci. 37, 9 (2002) 1801-1807.

[21] J. E. Zorzi, C. A. Perottoni, J. A. H. da Jornada, J. Mater. Sci. Lett. 22, 2 (2003) 107-109.

[22] J. E. Zorzi, C. A. Perottoni, J. A. H. da Jornada, Mater. Lett. 57, 24-25 (2003) 3784-3788.

[23] H. A. Barnes, "A Handbook of Elementary Rheology", Univ. Wales Institute of Non-Newtonian Fluid Mechanics, 
Dept. Mathematics, University of Wales Aberystwyth (2000).

[24] G. Schramm, "A Practical Approach to Rheology and Rheometry", $2^{\text {nd }}$ ed., Kalsruhe, Gebrueder HAAKE GmbH, Alemanha (1998).
[25] C. W. Macosko, "Rheology Principles, Measurements, and Applications", Wiley-VCH, New York, EUA (1994).

[26] R. E. F. Q. Nogueira, A. C. Bezerra, F. C. dos Santos, M. R. de Souza, W. Acchar, Key Eng. Mater. 189-191 (2001) 67-72.

(Rec. 25/06/2014, Rev. 30/01/2015, Ac. 31/01/2015) 